\title{
Cannabis: efectos nocivos sobre la salud mental
}

\author{
Quiroga, M. \\ Servicio de Psiquiatría. Hospital Militar "Pagés". Melilla. España \\ Teniente Coronel Médico Especialista en Psiquiatría, Jefe del Servicio de Psiquiatría
}

Enviar correspondencia a: Manuel Quiroga Gallego. Teniente Coronel Médico Psiquiatra. Servicio de Psiquiatría. Hospital Militar "Pagés". C/ General Polavieja s/n, 52071 Melilla (España) Tf.: 9526711 45, Extensión 236 Correo electrónico: mquirogag.intersep.org

\section{Resumen}

El consumo prolongado de THC puede producir un síndrome de dependencia donde los factores genéticos son más importantes que los ambientales.

La interrupción del consumo continuado puede provocar un síndrome de abstinencia caracterizado por ansiedad, tensión, desazón, alteraciones del sueño y cambios del apetito, mediado por agonistas endógenos y receptores cannabinoides cerebrales específicos.

EITHC puede causar episodios psicóticos agudos sin alteración del nivel de conciencia que son diferenciables de las psicosis. No se ha podido establecer una psicosis persistente por acción exclusiva del cannabis, pues sigue sin determinarse si el THC es detonante, agravante, mero acompañante o una forma de automedicación de un trastorno mental primario.

El consumo prolongado de dosis altas de THC puede conducir a un estado de pasividad e indiferencia (síndrome amotivacional), del que empieza a haber evidencias neuropsicológicas, morfológicas y funcionales.

El consumo precoz y repetido de cannabis en sujetos genéticamente predispuestos, puede estar relacionado con esquizofrenias cuyo primer episodio aparece tras un año de fumar THC, presentan más síntomas positivos y menos negativos, responden peor a los antipsicóticos y tienen más recaídas en los siguientes 15 años que los esquizofrénicos no consumidores. Ello parece ser debido a que en los esquizofrénicos se produce una alteración del sistema cannabinoide endógeno similar a la originada por la intoxicación cannábica en sujetos sanos.

En los adolescentes, iniciarse precozmente en el consumo de cannabis parece tener relación con agresividad, involucrarse en actividades delictivas, menospreciar el peligro de utilizar otras drogas y descuidar la protección del SIDA.

Palabras clave: cannabis, trastorno mental orgánico, psicosis, sindrome amotivacional, esquizofrenia, dependencia, síndrome de abstinencia

\section{Summary}

The continuous THC consumption is related to a dependency syndrome, where the genetic factors are more important than the environmental. Some times we find also a withdrawal syndrome characterized by anxiety, tension, sleep and food disorders, mediated by endogenous cannabinoids agonist and its specific cerebral receptor. There are acute psychotic episodes produced by cannabis, which must be differentiated from functional psychosis. It has not been possible to establish a persistent psychosis exclusively produced by cannabis, because its role is not still clear in relation to such psychosis.

The extended consumption of high dose of $\mathrm{THC}$ can lead to a passiveness and nonchalance state (amotivational syndrome), which begins to have neuropsychologicals, morphologics and functionals evidences.

The early and repeated consumption of cannabis in some subjects genetically predisposed, results in schizophrenia whose first episode appears after smoking THC one year, with more positive psychotic symptoms, responding worse to the antipsychotic medication and with more relapses in the following 15 years than other schizophrenics not using cannabis. This relation can be due to the fact that the schizophrenia produces an alteration of the endogenous cannabinoid system similar to the changes produced by cannabis intoxication in healthy subjects.

The cannabis early consumption in adolescents is related to aggressive behavior, to antisocial activities, to undervalue the danger of using other drugs and to neglect the protection of AIDS.

Key words: cannabis, mental disorder, psychosis, schizophrenia, amotivational syndrome, dependence, withdrawal syndrome. 


\section{INTRODUCCIÓN}

E responsable directo de los efectos psíquicos del cannabis es el D9 Tetrahidrocannabinol (THC), cuya concentración en las diferentes preparaciones usadas ("porro", "marihuana", "kiffi," "bhang," "dagga", "ganja", "dawamesc") varía según el tipo de planta y la zona geográfica de cultivo (1).

La primera publicación científica en occidente sobre los efectos psíquicos del THC, "Du haschich et de I'aliénation mental" (1845), se debe a Jacques-Joseph Moreau (1804-1884), médico francés del Hospital Psiquiátrico de La Bicêtre (París), y es fruto de la minuciosa observación del estado que él mismo y sus colaboradores alcanzaban con la ingestión de diferentes dosis de un preparado de cannabis elaborado por el propio Moreau (2). En ella se refieren 8 síntomas que compartían la intoxicación cannábica y las enfermedades mentales (alienación) de la época: euforia, excitación intelectual con disociación de las ideas, distorsión de la percepción del espacio y del tiempo, hipersensibilidad auditiva, ideas fijas y convicciones delirantes, inestabilidad de las emociones, impulsos irresistibles, ilusiones y alucinaciones $(2,3)$ que son dosis dependientes, sin menospreciar la susceptibilidad individual (3).

Otro psiquiatra, Walter Bromberg, americano, basado en su experiencia personal y en multitud de observaciones, describe en 1934, ochenta y nueve años después que Moreau, los efectos psíquicos agudos de la marihuana (cigarrillo de cannabis), cifrando la persistencia de la intoxicación en dos a cuatro horas tras fumarla y en cinco a doce cuando es ingerida (4). Los síntomas descritos por Bromberg, en otro lenguaje, son superponibles a los referidos por Moreau y compartidos por la mayoría de los observadores, con la reserva de que pueden variar ampliamente $y$, generalmente, son menos intensos (5).

Hace 25 años, aproximadamente, los efectos inmediatos directos del THC sobre la salud mental se podían resumir en que, además de los estados de intoxicación aguda (10 a $15 \mathrm{mg}$ de THC fumado; 20 a $40 \mathrm{mg}$ de THC ingerido), su consumo modificaba la presentación de los síntomas en algunas enfermedades mentales (patoplastia), inducía reacciones ansioso-depresivas cuando el consumidor se encontraba en situaciones desfavorables y podía precipitar el inicio de una psicosis latente, en tanto que su uso intenso y prolongado (100 a 300 mg/día) producía en los adultos deterioro mental y físico y en los adolescentes impedía la adecuada maduración de la personalidad (2).

En las más recientes clasificaciones internacionales de los trastornos mentales $(6,7)$ se recogen diversos cuadros psiquiátricos inducidos por cannabis (intoxicación, delirium, alucinatorio), poco específicos pues comparten criterios diagnósticos con otras drogas de naturaleza muy diferentes al THC.

Desde el año 1997, la Organización Mundial de la Salud (OMS) considera que el cannabis incide negativamente en la salud mental porque parece ser capaz de ocasionar un síndrome de dependencia, similar al de otras drogas, inducir psicosis propias, causar un síndrome amotivacional, y precipitar el inicio y exacerbar el curso de la esquizofrenia (8).

\section{OBJETIVOS}

El presente capítulo se centra en los efectos nocivos indicados por la OMS, revisando el estado actual de conocimientos y buscando los acuerdos y divergencias que sobre ellos pueda haber.

Finalmente, se reflejarán las principales líneas sobre las que la OMS considera debe proseguir la investigación de los efectos nocivos del cannabis sobre la salud mental.

\section{METODOLOGÍA}

Partiendo de los principales hitos históricos en el ámbito occidental sobre los efectos psicopatológicos del cannabis, se revisa la litera- 
tura profesional en torno a los cuatro efectos nocivos principales que para la OMS tiene el cannabis sobre la salud mental.

La exposición, en un intento de ser didáctica, los abordará por separado, aunque esta disección contradiga la realidad clínica.

Por último, se sintetizan en sendas tablas aquellos efectos que demuestren mayor evidencia por consenso de los estudios realizados.

La búsqueda de referencias bibliográficas se ha realizado en MEDLINE® mediante Eva-

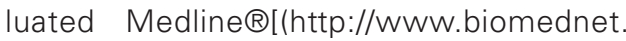
com/db/medline) (http://www.research.bmn. com/medline)]

\section{¿ PRODUCE SÍNDROME DE DEPENDENCIA EL CANNABIS?}

Hasta no hace mucho tiempo, y aún hoy, la gente y muchos profesionales sanitarios, creían que el THC no producía dependencia física con su consecuente síndrome de abstinencia, situación todavía reflejada en el DSM-IV ${ }^{\circledR}(9)$.

Sin embargo, hace ya 20 años, estudios en animales y humanos demostraron efectos leves de tolerancia y que la supresión del consumo de THC se acompaña de un conjunto de síntomas característicos, de duración media de 8 días (3). Por tanto, si hay tolerancia y abstinencia, el cannabis produce dependencia física.

Más próximos a nuestros días, uno de los estudios más representativos de la existencia de un síndrome de abstinencia debido al THC es el realizado sobre 5611 hombres y mujeres que fueron objeto de investigación entre 1991 y1995, y a los que se aplicaron los criterios diagnósticos estándar para el síndrome de dependencia, comprobándose que entre quienes habían consumido THC diariamente durante 70 meses o más, presentaban un síndrome de abstinencia característico: ansiedad, tensión, desazón, alteraciones del sueño y cambios en el apetito (10).

En adolescentes, 165 chicos y 64 chicas, entre 13 a 19 años, admitidos a tratamiento por adicción al cannabis, dos tercios presentaron síndrome de abstinencia (11).

Una de las más actuales revisiones sobre la farmacología, posibilidades terapéuticas, efectos adversos, e implicaciones sociales del uso médico de la marihuana (THC) confirma que tiene poder adictivo y produce síndrome de abstinencia, en menor grado que el alcohol, la cocaína, la heroína y la nicotina (12).

Las sustancias endógenas de efectos similares al THC (anandamida) y los receptores cannabinoides específicos (CB1) en el sistema nervioso central, al igual que sucede para la morfina y la nicotina, son las bases biológicas de la dependencia y la abstinencia del cannabis (13).

Desde el punto de vista experimental, en 1999 se publican dos trabajos $(14,15)$ que demuestran que el consumo intermitente de $\mathrm{THC}$ a dosis crecientes genera síntomas de abstinencia que difieren según sea fumado o ingerido por vía oral. En los fumadores, los síntomas de abstinencia que se encontraron fueron ansiedad, irritabilidad, gastralgia y disminución del apetito en relación con el que tenían antes del experimento (14). El síndrome de abstinencia debido al consumo de THC ingerido, presentaba ansiedad, depresión, irritabilidad, alteraciones en la calidad y cantidad del sueño y disminución del apetito (15)

De estos últimos trabajos se deduce que una de las causas para seguir consumiendo THC es evitar la aparición del síndrome de abstinencia.

Estudios con parejas de gemelos de ambos sexos, mono y dicigóticos, están demostrando que en el desarrollo de la dependencia del cannabis son más importantes los factores genéticos que los ambientales (16-18).

\section{¿INDUCE EL CANNABIS PSICOSIS PRO- PIAS?}

Lo primero que hay que determinar, ponerse de acuerdo, al afrontar este apartado es qué entendemos por psicosis inducidas, causadas, debidas, al cannabis. 


\begin{tabular}{|c|c|c|c|}
\hline $\begin{array}{l}\text { Año } \\
\text { estudio }\end{array}$ & Metodología & Autor & Ref. $n^{\circ}$ \\
\hline 1996 & $\begin{array}{l}\text { entrevista semiestructurada, cara a cara, aplicando criterios diagnósticos estándares } \\
\text { en } 5611 \text { hombre y mujeres }\end{array}$ & Wiesbeck et al. & 10 \\
\hline 1998 & $\begin{array}{l}\text { escalas de diagnóstico para dependencia de sustancias, trastornos mentales y p } \\
\left.\text { atrones de abuso de sustancias en adolescentes ( } 165_{-} \text {y } 64 \_\right)\end{array}$ & Crowley et al. & 11 \\
\hline 1998 & $\begin{array}{l}\text { revisión bibliográfica de la farmacología, terapéutica, efectos adversos e implicaciones } \\
\text { sociales del uso médico del THC }\end{array}$ & Taylor & 12 \\
\hline 1999 & $\begin{array}{l}\text { revisión bibliográfica sobre los efectos neurotóxicos del THC, morfina y nicotina, y de } \\
\text { sus respectivos agonistas endógenos y recep-tores específicos }\end{array}$ & Kobayashi et al. & 13 \\
\hline 1999 & síntomas de abstinencia tras fumar THC en 12 sujetos & Haney et al. & 14 \\
\hline 1999 & síntomas de abstinencia tras consumo oral de THC en $6_{-}$y 6 _ & Haney et al. & 15 \\
\hline
\end{tabular}

Bajo tal término, podemos encontrar referencias a trastornos agudos debidos al consumo de altas dosis de THC o como respuestas idiosincrásicas patológicas a bajas dosis; también, trastornos mentales de varias semanas de duración que se originaron consumiendo THC y han persistido después de dejar de consumir; incluso, se han incluido alteraciones del humor y cognitivas que se atribuían a haber consumido dosis muy altas de THC durante mucho tiempo.

Desde una perspectiva práctica, y con los inconvenientes de las simplificaciones, en este capítulo nos referiremos a: 1) trastornos mentales orgánicos, esto es, alteraciones psicopatológicas agudas debidas a la acción inmediata del THC; 2) aparición de episodios psicóticos sin alteración del nivel de conciencia con ocasión del consumo del THC; 3) estados psicóticos persistentes tras interrumpir el consumo de THC (psicosis cannábica) que tengan una clínica y evolución que permitan diferenciarlas de otras psicosis funcionales.

1) Trastornos mentales orgánicos.

Existe acuerdo universal $(9,16)$ en que el THC causa trastornos mentales orgánicos (intoxicación, delirium, alucinatorio), más allá de los efectos que el consumidor busca como propios de la embriaguez cannábica, resultado directo de la concentración de la sustancia en el cerebro y de la sensibilidad particular de éste hacia aquella.

Además, los síntomas asociados al consumo de cualquier sustancia psicotropa, legal o ilegal, y también el cannabis, pueden imitar todas las formas de psicopatología primaria (20).

2) Aparición de episodios psicóticos sin alteración del nivel de conciencia.

Los trastornos psicóticos que aparecen directamente relacionados con el consumo de cannabis, sin disminución del nivel de conciencia, presentan angustia, humor inestable de aspecto hipomaníaco, autolesiones, conducta desorganizada y hostilidad, suspicacia, ideación paranoide, aceleración del pensamiento y fuga de ideas, sentimientos de grandiosidad, alucinaciones no verbales (2129).

Metodológicamente, algunos de los estudios anteriores pueden ser discutidos pues no se basan en el estudio controlado de 


\begin{tabular}{|l|l|l|l|}
\hline \multicolumn{3}{|c|}{ Tabla 2. Episodios psicóticos con conciencia lúcida } \\
\hline $\begin{array}{c}\text { Año } \\
\text { estudio }\end{array}$ & \multicolumn{1}{|c|}{ Metodología' } & Autor & Ref.n $^{\circ}$ \\
\hline 1976 & 25 psicosis por cannabis vs 25 esquizofrénicos paranoides & Thacore et al. & 22 \\
\hline 1982 & 20 psicóticos THC positivo en orina vs 20 psicóticos libres de cannabis & Rottanburg et al. & 23 \\
\hline 1991 & síntomas ingestión bhang en no psicóticos vs síntomas ingestión bhang en psicóticos & Cahudry et al. & 26 \\
\hline 1992 & $\begin{array}{l}\text { psicóticos THC positivos en orina vs psicóticos sin THC, comparando síntomas a la } \\
\text { semana, mes y seis meses }\end{array}$ & Mathers et al. & 27 \\
\hline 1999 & $\begin{array}{l}20 \text { casos de psicosis por cannabis vs } 20 \text { casos 'episodio esquizofrénico agudo' } \\
\text { 'Sólo estudios con control de casos }\end{array}$ & Basu et al. & 30 \\
\hline
\end{tabular}

casos, esto es, comparar la psicopatología y el curso del episodio entre sujetos con orinas positivas a cannabinoides frente a negativas (8).

En estudios realizados mediante control de casos, los episodios psicóticos debidos al cannabis en comparación con los controles libres de THC en orina, presentaban más conductas desorganizadas y violentas con pánico y menos alteraciones formales del pensamiento (22); la evolución era más breve y con manifiestos aspectos hipomaníacos (23); revisten el aspecto de un desorden maniforme con elementos paranoides y disfunción cognitiva (26); las diferencias en síntomas al ingreso desaparecían al cabo de una semana (27); tenían menos alteraciones formales del pensamiento, al afectividad era reactiva y congruente, predomina el polimorfismo en los síntomas clínicos, con corta duración y a las recaídas les precedía siempre el consumo de cannabis (30).

3) Estados psicóticos persistentes (psicosis cannábica).

La controversia (31), de si el consumo prolongado de dosis elevadas THC (tasa en sangre mayor de $15 \mathrm{mg}$ ) origina psicosis típicas (cannabis-induced psychosis) o se trata del primer episodio de una psicosis funcional (esquizofrénica o afectiva) coincidente con la intoxicación cannábica, está aún vigente (8, 32-34).

Estudios realizados en diferentes zonas geoeconómicas y culturales, tales como Tailandia (35), Jamaica (36), Inglaterra $(37,40)$, Sudáfrica (38), Nigeria (39), Dinamarca (41) concluyen que los estados psicóticos repetidos en consumidores crónicos de cannabis se presentan principalmente como esquizofrénicos o maníacos y corresponden a una exacerbación y/o modificación patoplástica de la psicosis primaria, con tendencia a la recidiva si se persiste en el consumo de THC.

En un estudio, realizado en Inglaterra y publicado en 1994, comparando 23 psicóticos con THC positivo en orina frente a 46 psicóticos libres de droga, concluía que las psicosis que se desarrollan o recurren en el contexto del uso del cannabis no tienen características psicopatológicas o forma de inicio, ni están restringidas a grupos étnicos, ni sociodemográficos que las distingan de los controles (42).

Recientemente, en Alemania, se han descrito dos casos de psicosis con intensas alteraciones formales del pensamiento y de la percepción, delirio y afectividad restringida que se presentaron en ausencia de antecedentes psicóticos, tras consumir continuamente THC durante 8 y 18 meses, respectivamente (43). 


\begin{tabular}{|c|c|c|c|}
\hline $\begin{array}{l}\text { Año } \\
\text { publicac. }\end{array}$ & Metodología & Autor & Ref. $n^{\circ}$ \\
\hline 1994 & 23 psicóticos THC positivos orina vs 46 psicóticos libres THC, no existen diferencias & McGuire et al. & 42 \\
\hline 1999 & $\begin{array}{l}\text { dos casos de psicosis graves tras consumir } 8 \text { y } 18 \text { meses THC, sin antecedentes } \\
\text { psicóticos }\end{array}$ & Leweke et al & 43 \\
\hline
\end{tabular}

¿OCASIONA SÍNDROME AMOTIVACIONAL?

Por 'síndrome amotivacional' se entiende un estado de pasividad e indiferencia, caracterizado por disfunción generalizada de las capacidades cognitivas, interpersonales y sociales (3) debido al consumo de cannabis durante años y que persistiría una vez interrumpido dicho consumo.

Para la OMS, sigue sin estar suficientemente diferenciado este "síndrome amotivacional" de los efectos directos de la intoxicación crónica por THC (8).

Comparando consumidores diarios durante años (6 años de media) con otros que lo hacía varias veces al mes durante menos tiempo (4.5 años de media), parece ser que los síntomas del síndrome amotivacional encontrados en el primer grupo corresponden a una depresión (44).

Hay autores que consideran que el síndrome amotivacional no sólo lo causa el consumo prolongado de altas dosis de THC, sino también, las anfetaminas, la cocaína y los solventes volátiles orgánicos (45).

Y es precisamente en usuarios crónicos de solventes orgánicos volátiles, que se han descrito alteraciones del flujo cerebral, medido con tomografía computarizada por emisión única de fotones (SPECT), en el córtex frontal de ambos hemisferios cerebrales correlacionando la intensidad de la disminución del flujo con el incremento de la puntuación en la escala "abolición-apatía" del SANS (Scale for the Assessment of Negative
Symptoms) (46). También se ha encontrado esta correlación entre la disfunción frontal identificada mediante examen neuropsicológico y la hipoperfusión del córtex de los lóbulos frontales especialmente en su zona medial (47).

Respecto al cannabis, su consumo repetido ha demostrado que reduce el flujo cerebral (SPECT) en ambos lóbulos temporales (48) y que altera el trazado del electroencefalograma (EEG) de forma tal que en consumidores diarios de THC entre 15 a 24 años en comparación con no consumidores y consumidores durante menos tiempo, se encontró que, además de la hiperfrontalidad alfa, la actividad theta se alteraba bilateralmente en la región fronto-central, al tiempo que incrementaba su coherencia interhemisférica en zonas centrales y posteriores (49), con marcada reducción de la actividad alfa en aquellos que habían estado consumiendo diariamente durante más años (50).

Quizás, continuar investigando correspondencias entre síntomas negativos y variaciones regionales del flujo sanguíneo cerebral y en la actividad bioeléctrica cerebral, sea el camino para dilucidar si realmente existe un síndrome amotivacional inducido por cannabis.

\section{CANNABIS Y ESQUIZOFRENIA}

La OMS admite que el consumo persistente de THC en relación con la esquizofrenia (8) precipita su inicio, sobre todo en quienes empe- 


\begin{tabular}{|l|c|c|c|}
\hline \multicolumn{2}{|c|}{ Tabla 4. Síndrome amotivacional' } & Autor & Ref.n \\
\hline $\begin{array}{c}\text { Año } \\
\text { publicac. }\end{array}$ & \multicolumn{1}{|c|}{ Metodología } & Musty et al & 44 \\
\hline 1995 & THC diario 6 años vs 4,5 años: síntomas primer grupo es una depresión & Okada et al. & 48 \\
\hline 1999 & THC reduce flujo sanguíneo cerebral en ambos lóbulos temporales & Struve et al & 50 \\
\hline 1999 & $\begin{array}{l}\text { THC consumo crónico altera EEG: hiperfontalidad alfa, hipersincronía theta bilateral, } \\
\text { reducción frecuencia alfa }\end{array}$ & & \\
\hline
\end{tabular}

zaron con el cannabis antes de los 18 años, aumenta el número de recaídas en los siguientes 15 años y la gravedad de las mismas (5153) reconociendo que ello es más factible cuando el THC se consume sobre una predisposición, vulnerabilidad, esquizofrénica (35, 54-57) y, quizás también, por la falta de tratamiento adecuado para sus dos patología, la de su dependencia y la de su esquizofrenia (53).

Actualmente, la predisposición o vulnerabilidad se está considerando, como el resultado de la interacción de la organización genética personal con factores de riesgo ambiental, entre los que se incluye el cannabis $(58,59)$.

El mecanismo por el que se produce en consumidores de THC el inicio precoz y mayor número de recaídas en las psicosis esquizofrénicas comienza a ser explicado sobre la base de que el sistema de recepto- res cannabinoides cerebrales y sus activadores endógenos se encuentran alterados en esquizofrénicos no consumidores de THC.

En este sentido, se ha demostrado que la intoxicación cannábica en voluntarios sanos produce alteraciones similares a las observadas en pacientes esquizofrénicos no consumidores de THC en pruebas de visión estereoscópica $(60,61)$.

Más recientemente, comparando la concentración de cannabinoides endógenos (anandamida y plamitiletanolamida) en líquido cefalorraquídeo de 10 pacientes con esquizofrenia con 11 no esquizofrénicos (controles), se encontraron niveles significativamente mayores en el grupo de esquizofrénicos que en los controles, sin que estos resultados estuvieran condicionados ni por el sexo, ni por la edad ni por la medicación antipsicótica (62).

\begin{tabular}{|c|c|c|c|}
\hline \multicolumn{4}{|c|}{ Tabla 5. Cannabis y esquizofrenia } \\
\hline $\begin{array}{l}\text { Año } \\
\text { publicac. }\end{array}$ & Metodología & Autor & Ref. $n^{\circ}$ \\
\hline 1999 & $\begin{array}{l}27 / 39 \text { esquizofrénicos con historia de abuso de THC actual vs } 26 / 39 \text { grupo control no } \\
\text { usuario THC, fueron seguidos } 68.7+28.3 \text { meses }\end{array}$ & Caspari et al. & 52 \\
\hline 1998 & $\begin{array}{l}\text { en pruebas visión estereoscópica, intoxicación THC en voluntarios sanos causa altera- } \\
\text { ciones similares a las observadas en esquizofrénicos no consumidores THC }\end{array}$ & Schneider et al & 61 \\
\hline 1999 & $\begin{array}{l}\text { Cannabinoides endógenos en LCR en } 10 \text { esquizofrénicos libres drogas concentración } \\
\text { mayor que } 11 \text { no esquizofrénicos controles }\end{array}$ & Leweke et al. & 62 \\
\hline
\end{tabular}




\section{OTROSTRASTORNOS}

Para la OMS, el trastorno amnésico está suficientemente demostrado, en tanto que la despersonalización y los flash-back (revivir una intoxicación sin haber consumido THC) se sustentan en algunas descripciones de casos, sin poder afirmar que el cannabis, y no otras drogas, sea el único responsable de estos dos cuadros(8).

Se han descrito casos de intoxicación con síntomas gastrointestinales y psicológicos tras comer ensalada aliñada con aceite de semillas de cáñamo (63).

El seguimiento de consumidores de THC desde el inicio de la adolescencia demostró que 5 años después abandonaban los estudios, no percibían peligro en otras drogas, menospreciaban el uso de preservativos en las relaciones sexuales, con el consiguiente incremento del riesgo para el SIDA y se involucraban en actividades delictivas (64).

Un problema particular lo plantea la relación entre cannabis y la agresividad, estrechamente vinculados antiguamente (años 1090 al 1260) hasta el punto de que de esa creencia se hace proceder la palabra asesino (65).

En un experimento en el que los sujetos fumaban placebo o cigarrillos de $\mathrm{THC}$ con tres diferentes concentraciones, se evidenció que las respuestas agresivas incrementaban en la primera hora después de fumar THC y no descendían al nivel del placebo hasta un día después (66).

Inversamente, la agresividad preadolescente es un factor de riesgo para el consumo de cannabis, alcohol, otras drogas y conductas delictivas en la adolescencia (67), que hace que los chicos se decanten por el THC y las drogas mucho más que las chicas, diferencia que no se observa para el alcohol (68).

En el año 1999 se publica un estudio sobre la conducta agresiva durante el síndrome de abstinencia en sujetos que habían fumado cannabis en al menos 5.000 ocasiones (el equivalente a un "porro" diario durante casi 14 años), encontrándose que, en compara- ción con los niveles pre-abstinencia, la agresividad aumentaba desde el día 3 al 28 tras la supresión delTHC (69).

En la práctica forense, tres aspectos son importantes: 1) determinar la concentración de THC en sangre capaz de producir efectos psicofísicos subjetivos; 2) dilucidar hasta qué punto el THC es el factor más importante en la aparición de una conducta; y, 3) las repercusiones médicolegales de la inhalación pasiva del humo del cannabis.

Hoy en día existen modelos farmacodinámicos (70) y de predicción de exposición al THC (71) que han permitido concluir que para experimentar un $50 \%$ de los efectos subjetivos más intensos se precisan concentraciones plasmáticas de THC entre 7 y $29 \mathrm{ng} / \mathrm{ml}$ (72). A título de ejemplo, el Departamento de Defensa americano ha fijado el límite de 15 $\mathrm{ng} / \mathrm{ml}$ de 11-nor-delta-tetrahidrocannabinol-9carboxílico (THCA) en orina como la concentración que se considera no punible militarmente (73).

Sigue siendo un tema controvertido el dilucidar hasta qué punto es el cannabis el responsable, principal o exclusivo, de una conducta ilícita durante un episodio psicótico (74, 75), así como su importancia en las muertes violentas en jóvenes (76-78).

La inhalación pasiva de humo de cannabis, que supondría la involuntaria intoxicación del sujeto y, por tanto, su falta de responsabilidad penal, ha planteado la necesidad de investigar modelos que permitan relacionar su presencia en el aire en el interior de locales con la concentración urinaria del THCA (73).

\section{LINEAS PARA PROSEGUIR LA INVESTIGA- CION}

Delimitar mediante técnicas neurorradiológicas y electroencefalográficas el daño cerebral que pueda causar el consumo crónico, y, con ello, las características morfofuncionales y clínicas del síndrome amotivacional. 
Delimitar con estudios controlados de casos los síntomas de la dependencia y de la abstinencia, incluyendo medidas de tratamiento y reinserción.

Diseños experimentales bien controlados y estudios de casos-control para identificar clínica y evolutivamente las psicosis agudas y/o crónicas debidas al cannabis.

Estudios de seguimiento prolongado de casos de esquizofrenia que se iniciaron consumiendo cannabis para ver cómo la supresión del THC condiciona la evolución.

Profundizar en las alteraciones del sistema cannabinoide endógeno tanto en estados de intoxicación en voluntarios sanos, como en esquizofrénicos consumidores y no consumidores de cannabis.

\section{CONCLUSIONES}

El cannabis, la droga ilegal más utilizada por la población joven y la que más precozmente se empieza a consumir, causa efectos nocivos agudos y crónicos sobre la salud mental.

El consumo prolongado de THC produce un síndrome de dependencia (tolerancia, con necesidad de proseguir el consumo para evitar la abstinencia), en el que los factores genéticos son más importantes que los ambientales.

La interrupción del consumo continuado de cannabis provoca un síndrome de abstinencia caracterizado por ansiedad, tensión, desazón, alteraciones del sueño y cambios del apetito, con discretas diferencias en la intensidad y duración de los síntomas según que el THC se haya fumado o ingerido, mediado por agonistas endógenos (anandamida) y receptores cannabinoides cerebrales específicos (CB1).

EITHC produce trastornos mentales orgánicos, esto es, estados de inicio brusco y duración de horas, no necesariamente dosis dependientes, que exceden de los efectos buscados por el consumidor.
Se presentan episodios psicóticos agudos sin alteración del nivel de conciencia que se distinguen de las psicosis funcionales porque tienen menos alteraciones formales del pensamiento, la afectividad suele reactiva, congruente y tomar un aspecto maniforme, la conducta es a menudo hostil, la duración es breve y su reaparición siempre va precedida del consumo de THC.

Sin embargo, no se ha podido establecer todavía una psicosis persistente por acción exclusiva del cannabis, pues sigue sin determinarse si el cannabis es el detonante, un agravante, mero acompañante o una forma de automedicación de un trastorno mental primario. $(53,79)$.

El consumo de dosis altas de THC durante mucho tiempo conduce a un estado de pasividad e indiferencia caracterizado por disfunción generalizada de las capacidades cognitivas, interpersonales y sociales (llamado síndrome amotivacional) del que empieza a haber evidencias neuropsicológicas, morfológicas y funcionales.

El consumo repetido y precoz de cannabis en sujetos genéticamente predispuestos, sobre todo cuando coinciden la predisposición para depender de la droga y para la esquizofrenia, resulta en esquizofrenias cuyo primer episodio aparece tras un año de consumo de THC, generalmente antes de los 18 años, que presentan más síntomas positivos y menos negativos, con peor respuesta a los antipsicóticos, y más recaídas en los siguientes 15 años que los esquizofrénicos no consumidores.

La razón de esta particular influencia del THC en la esquizofrenia parece ser debida a que en los esquizofrénicos se produce una alteración del sistema cannabinoide endógeno similar a la originada por la intoxicación cannábica en sujetos sanos.

En los adolescentes, iniciar precozmente el consumo de cannabis tiene como efectos nocivos peculiares a corto plazo menospreciar el peligro de utilizar otras drogas, descuidar la protección del SIDA, la agresividad e involucrarse en actividades delictivas. 
En los jóvenes, el consumo de cannabis complica mucho la valoración psiquiátrico forense en aquellas conductas psicóticas que suceden en el marco de un uso reiterado, $y$, además es un factor de riesgo de muerte violenta.

Un aspecto novedoso es la creciente importancia medicolegal de la inhalación pasiva del humo del THC.

\section{BIBLIOGRAFÍA}

Beneit Montesinos JV, Portoles Pérez A, Lizasoain Hernández I. Características químicas y farmacocinéticas de los cannabinoides. En Beneit Montesinos JV, López Corral JC, editores de Drogodependencias. Aspectos famacológicos y clínicos, Escuela Universitaria de Enfermería, Fisioterapia y Podología, Universidad Complutense de Madrid, 1990:167-81.

Nahas G. Haschis, cannabis et marijuana. Premièr Edition, Vendôme (France), Presses Universitaires de Fance, 1976:243-95.

Gold MS. Marihuana Ediciones en neurociencias, Primera Edición, Barcelona, 1991:91-101.

Grinspoon L. Dependencia de las drogas: agentes no narcóticos. En Freedman AM, Kaplan HI, Sadock BJ, editores de Tratado de psiquiatría, Salvat Editores S.A., Barcelona 1982;II (23.2): 1467-81.

Grinspoon L, Bakalar JB. Drogodependencia: agentes no narcóticos. En Kaplan HI, Sadock BJ, Cancro R, directores de Tratado de Psiquiatría, Segunda Edición. Salvat Editores S.A., Barcelona 1989:II(22.2):995-1007.

Organización Mundial de la Salud. F10-F19 "Trastornos mentales y del comportamiento debidos al consumo de sustancias psicotropas". En Décima revisión de la Clasificación Internacional de Enfermedades. Trastornos mentales y del comportamiento. Descripciones clínicas y pautas para el diagnóstico. Meditor y JJ López Ibor, Madrid 1992:97-112.

American Psychiatric Association. Trastornos relacionados con Cannabis. En DSM-V. Manual diagnóstico y estadístico de los trastornos mentales. Cuarta Revisión. Edición española Salvat Editores S.A. Barcelona 1995:227-33.
Division of mental health and prevention of substance abuse. World Health Organization. Cannabis: a health perspective and research agenda. WHO/MSA/PSA/97.4 Distr.: General. Geneva 1997.

Frances RJ, Franklin JE. Trastornos por uso del alcohol y otras sustancias psicoactivas. En Hales RE, Yudofsky SC, Talbot JA, Directores de Tratado de Psiquiatría, Segunda Edición, Ancora S.A., Barcelona, 1996;11:373-434.

Wiesbeck GA, Schuckit MA, Kalmijn JA, Tipp JE, Bucholz KK, Smith TL. An Evaluation of the History of a Marijuana Withdrawal Syndrome in a Large Population. Addiction 1996;91(10):14691478.

Crowley TJ, Macdonald MJ, Whitmore EA, Mikulich, SK. Cannabis dependence, withdrawal, and reinforcing effects among adolescents with conduct symptoms and substance use disorders. Drug Alcohol Depend 1998;50(1):2737.

Taylor HG. Analysis of the medical use of marijuana and its societal implications. J Am Pharm Assoc (Wash) 1998;38(2):220-7.

Kobayashi H, Suzuki T, Kamata R, Saito S, Sato I, Tsuda S, Matsusaka N. Recent progress in the neurotoxicology of natural drugs associated with dependence or addiction, their endogenous agonists and receptors. J Toxicol Sci 1999;24(1):1-16.

Haney M, Ward AS, Comer SD, Foltin RW, Fischman MW. Abstinence symptoms following smoked marijuana in humans. Psychopharmacology (Berl) 1999;141(4):395-404.

Haney M, Ward AS, Comer SD, Foltin RW, Fischman MW. Abstinence symptoms following oral THC administration to humans. Psychopharmacology (Berl) 1999;141(4):385-94.

Organización Mundial de la Salud. Libro de casos de la CIE-10: Las diversas caras de los trastornos mentales: Historias clínicas de casos de trastornos mentales y del comportamiento en personas adultas, comentadas según las descripciones de la CIE-10. Editorial Médica Panamericana S.A., Madrid 1999:62-3.

van den Bree MB, Johnson EO, Neale MC, Pickens RW. Genetic and environmental influences on drug use and abuse/dependence in male and female twins. Drug Alcohol Depend 1998;52 (3):231-41.

Kendler KS, Prescott CA. Cannabis use, abuse, and dependence in a population-based sample of 
female twins. Am J Psychiatry 1998;155(8): 1016-22.

Kendler KS, Karkowski LM, Neale MC, Prescott CA. Illicit psychoactive substance use, heavy use, abuse, and dependence in a US population-based sample of male twins. Arch Gen Psychiatry 2000;57(3):261-9.

Frances A, Ross R. DSM-IV(r) Estudio de casos. Guía clínica para el diagnóstico diferencial. Masson S.A. Barcelona 1999;55-68.

Farini Duggan J, Aust M. Marihuana psychoses. Acta Psiquiatr Psicol Am Lat 1976 Jan;22(1):6370.

Thacore VR, Shukla SR. Cannabis psychosis and paranoid schizophrenia. Arch Gen Psychiatry 1976 Mar;33(3):383-6.

Rottanburg D, Robins AH, Ben-Arie O, Teggin A, Elk R. Cannabis-associated psychosis with hypomanic features. Lancet 1982 Dec;2(8312):1364-6.

Thornicroft G. Cannabis and psychosis. Is there epidemiological evidence for an association?. $\mathrm{Br} \mathrm{J}$ Psychiatry 1990 Jul;157:25-33. [published erratum appears in Br J Psychiatry 1990 Sep;157: 460].

Crespo MD, Morales MP, Vinas R, Rios B, Ruiz L, Franco B. Psicosis y consumo de cannabis: estudio de las diferencias psicopatológicas y factores de riesgo. Actas Luso Esp Neurol Psiquiatr Cienc Afines 1990 Apr;18(2):120-4.

Chaudry HR, Moss HB, Bashir A, Suliman T Cannabis psychosis following bhang ingestion. $\mathrm{Br} J$ Addict 1991 Sep;86(9):1075-1081.

Mathers DC, Ghodse AH. Cannabis and psychotic illness. Br J Psychiatry 1992 Nov;161:648-53.

Quiroga Gallego M. Cannabis et psychopathologie parmis les jeunnes hommes. Types de troubles mentaux avec la cannabis positive dans l'urine. Europ Psychiatry 1996;11(4):327s.

Sembhi S, Lee JW. Cannabis use in psychotic patients. Aust N Z J Psychiatry 1999;33(4):52932.

Basu D, Malhotra A, Bhagat A, Varma VK. Cannabis psychosis and acute schizophrenia. a case-control study from India. Eur Addict Res 1999;5 (2):71-3.

Negrete JC. Effect of cannabis use on health. Acta Psiquiatr Psicol Am Lat 1983 Dec;29(4):267-76.

Court JM. Cannabis and brain function. J Paediatr Child Health 1998;34(1):1-5.
Campbell J. Cannabis: the evidence. Nurs Stand 1999;13(44):45-7.

Ungerleider JT. Marijuana: still a "signal of misunderstanding". Proc Assoc Am Physicians 1999; 111(2):173-81.

Kroll P. Psychoses associated with marijuana use in Thailand. J Nerv Ment Dis 1975 Sep;161(3): 149-56.

Knight F. Role of cannabis in psychiatric disturbance. Ann NY Acad Sci 1976;282:64-71.

Johnson BA. Psychopharmacological effects of cannabis. Br J Hosp Med 1990 Feb;43(2):114-6.

Solomons K, Neppe VM, Kuyl JM. Toxic cannabis psychosis is a valid entity. S Afr Med J 1990 Oct 20;78(8):476-81.

Imade AG, Ebie JC. A retrospective study of symptom patterns of cannabis-induced psychosis. Acta Psychiatr Scand 1991 Feb;83(2):134-6.

Thomas H. Psychiatric symptoms in cannabis users [see comments]. Br J Psychiatry 1993 Aug; 163:141-9.

Kristensen FW. Cannabis and psychoses. Ugeskr Laeger 1994 May;156(19):2875-8.

McGuire PK, Jones P, Harvey I, Bebbington P, Toone B, Lewis S, Murray RM Cannabis and acute psychosis. Schizophr Res 1994 Sep;13(2):161167.

Leweke FM, Emrich HM. Carbamazepine as an adjunct in the treatment of schizophrenia-like psychosis related to cannabis abuse. Int Clin Psychopharmacol 1999;14(1):37-9.

Musty RE, Kaback L. T Relationships between motivation and depression in chronic marijuana users Life Sci 1995; 56(23-34):2151-8.

Ashizawa T, Saito T, Yamamoto M, Shichinohe $S$, Ishikawa $\mathrm{H}$, Maeda $\mathrm{H}$ et al. [A case of amotivational syndrome as a residual symptom after methamphetamine abuse]. Nihon Arukoru Yakubutsu Igakkai Zasshi 1996;31(5):451-61.

Okada S, Yamanouchi N, Kodama K, Uchida Y, Hirai S, Sakamoto T, Noda S, Komatsu N, Sato T. Regional cerebral blood flow abnormalities in chronic solvent abusers. Psychiatry Clin Neurosci 1999;53(3):351-6.

Kitabayashi Y, Ueda H, Matsuda M, Narumoto J, Kobayashi T, Fukui K. [A case report of organic solvent abuse with amotivational syndrome]. Nihon Arukoru Yakubutsu Igakkai Zasshi 1999; 34(2):130-7. 
Amen DG, Waugh M. High resolution brain SPECT imaging of marijuana smokers with AD/HD. J Psychoactive Drugs 1998;30(2):209-14.

Struve FA, Patrick G, Straumanis JJ, Fitz-Gerald MJ, Manno J. Possible EEG sequelae of very long duration marihuana use: pilot findings from topographic quantitative EEG analyses of subjects with 15 to 24 years of cumulative daily exposure to THC. Clin Electroencephalogr 1998;29(1):31-6.

Struve F.A, Straumanis J.J, Patrick G, Leavitt J, Manno J.E, Manno B.R. Topographic quantitative EEG sequelae of chronic marihuana use: a replication using medically and psychiatrically screened normal subjects. Drug Alcohol Depend 1999;56(3):167-79.

van Amsterdam JG, van der Laan JW, Slangen JL. [Cognitive and psychotic effects after cessation of chronic cannabis use]. Ned Tijdschr Geneeskd 1998;142(10):504-8 (published erratum appears in Ned Tijdschr Geneeskd 1998 Mar 28;142(13):752).

Caspari D. Cannabis and schizophrenia: results of a follow-up study. Eur Arch Psychiatry Clin Neurosci 1999;249(1):45-9.

Rubio Valladolid G. Consumo de cannabis en diferentes grupos de población. En Cabrera Forneiro J, Editor de Cannabis ;Hasta dónde!. Agencia Antidroga, Consejería de Sanidad y Servicios Sociales, Comunidad de Madrid. Ediciones Harcourt, Madrid 1999;12:167-84.

Varma SL; Sharma I. Psychiatric morbidity in the first-degree relatives of schizophrenic patients. Br J Psychiatry 1993 May;162:672-8 [published erratum appears in $\mathrm{Br} J$ Psychiatry 1993 Nov; 163:700].

Hall W. The public health significance of cannabis use in Australia. Aust J Public Health 1995 Jun;19(3):235-42.

McGuire PK; Jones P; Harvey I; Williams M; McGuffin P; Murray RM. Morbid risk of schizophrenia for relatives of patients with cannabis-associated psychosis. Schizophr Res 1995 May;15(3)277-81.

Williams JH, Wellman NA, Rawlins JN. Cannabis use correlates with schizotypy in healthy people. Addiction 1996 Jun;91(6):869-77.

van Os J, Marcelis M. The ecogenetics of schizophrenia: a review. Schizophr Res 1998;32(2):12735.
Van Os J, Verdoux H. Aspects environnementaux et psychosociaux de la recherche genetique en psychiatrie. Encephale 1998;24(2):125-31.

Emrich HM, Leweke FM, Scneider V. Towards a cannabinoid hypotesis of schizophrenia: cognitive impairments due to dysregulation of the endogenous cannabinoid system. Pharmacol Biolchem Behav 1997;56:803-7.

Schneider U, Leweke FM, Mueller-Vahl KR, Emrich HM. Cannabinoid/anandamide system and schizophrenia: is there evidence for association?. Pharmacopsychiatry 1998;31(Suppl 2):110-3.

Leweke FM, Giuffrida A, Wurster U, Emrich HM, Piomelli D. Elevated endogenous cannabinoids in schizophrenia. Neuroreport 1999;10(8):1665-9.

Meier H, Vonesch HJ. [Cannabis poisoning after eating salad]. Schweiz Med Wochenschr 1997;127(6):214-8.

Brook J.S, Balka E.B, Whiteman M. The risks for late adolescence of early adolescent marijuana use. Am J Public Health 1999;89(10):1549-54.

Enciclopedia Universal Ilustrada Europeo-Americana. Espasa-Calpe, S.A., Madrid, 1909. Reimpresión de 1985. Tomo 6(ARD-AZZ):626-7.

Cherek DR, Roache JD, Egli M, Davis C, Spiga R, Cowan K. Acute effects of marijuana smoking on aggressive, escape and point-maintained responding of male drug users. Psychopharmacology (Berl) 1993;111(2):163-8.

Lochman JE, Wayland KK. Aggression, social acceptance, and race as predictors of negative adolescent outcomes. J Am Acad Child Adolesc Psychiatry 1994;33(7):1026-35.

Steele RG, Forehand R, Armistead L, Brody G. Predicting alcohol and drug use in early adulthood: the role of internalizing and externalizing behavior problems in early adolescence. Am J Orthopsychiatry 1995;65(3):380-8.

Kouri EM, Pope HG Jr, Lukas SE. Changes in aggressive behavior during withdrawal from long-term marijuana use. Psychopharmacology (Berl) 1999;143(3):302-8.

Huestis MA, Henningfield JE, Cone EJ. Blood cannabinoids. I. Absorption of THC and formation of 11-OH-THC and THCCOOH during and after smoking marijuana. J Anal Toxicol 1992 SepOct;16(5):276-82.

Huestis MA, Henningfield JE, Cone EJ. Blood cannabinoids. II. Models for the prediction of time of marijuana exposure from plasma concentra- 
tions of delta 9-tetrahydrocannabinol (THC) and 11-nor-9-carboxy-delta 9-tetrahydrocannabinol (THCCOOH). J Anal Toxicol 1992 Sep-Oct; 16(5): 283-90.

Cone EJ, Huestis MA Relating blood concentrations of tetrahydrocannabinol and metabolites to pharmacologic effects and time of marijuana usage. Ther Drug Monit 1993 Dec;15(6):52732.

Giardino NJ. An indoor air quality-pharmacokinetic simulation of passive inhalation of marijuana smoke and the resultant buildup of 11-nordelta-9-tetrahydrocannabinol-9-carboxylic acid in urine. J Forensic Sci 1997 Mar;42(2):323-5.

Hollweg M, Soyka M, Nedopil N. [Forensic psychiatric expert evaluation of so-called drug-induced psychoses] Psychiatr Prax 1995 Sep;22(5):197200.

Silva JA, Leong GB, Rhodes LJ, Weinstock R. A new variant of "subjective delusional" misiden- tification associated with aggression. J Forensic Sci 1997;42(3):406-10.

Marquet P, Delpla PA, Kerguelen S, Bremond J, Facy F, Garnier M, et al. Prevalence of drugs of abuse in urine of drivers involved in road accidents in France: a collaborative study. J Forensic Sci 1998;43(4):806-11.

Hansen AC, Kristensen IB, Dragsholt C Prevalence of cannabinoids in urine samples from forensic autopsies. Med Sci Law 1999 Jul;39(3):228-32.

Seymour A, Oliver JS Role of drugs and alcohol in impaired drivers and fatally injured drivers in the Strathclyde police region of Scotland, 19951998. Forensic Sci Int 1999 Jul 26;103(2):89100.

Krystal JH, D'Souza DC, Madonick S, Petrakis IL. Toward a rational pharmacotherapy of comorbid substance abuse in schizophrenic patients. Schizophr Res 1999;35(Supp 1):S35-49. 